Enfoques. Revista de Investigación en Ciencias de la Administración http://doi.org/10.33996/revistaenfoques.v5i17.106 enfoques No. 17 | Volumen 5 | Enero - marzo 2021 http://revistaenfoques.org ISSN: $2616-8219$ ISSN-L: $2616-8219$

\title{
EL NEUROMARKETING Y EL COMPORTAMIENTO DEL CONSUMIDOR DE CERVEZA
}

pp. $65-77$

\author{
NEUROMARKETING AND BEER CONSUMER BEHAVIOR
}

NEUROMARKETING E COMPORTAMENTO DO CONSUMIDOR DE CERVEJA

\section{Segovia Jaramillo Vladimir}

Artículo recibido octubre 2020 | Arbitrado noviembre 2020 | Publicado 01 de enero 2021

\begin{abstract}
Resumen
La aplicación del neuromarketing ha revolucionado el estudio del comportamiento del consumidor, generando beneficios para varios sectores, entre ellos la industria de la cerveza. El objetico de este estudio fue determinar el factor de influencia más relevante en el comportamiento de compra de los consumidores de cerveza en Sucre, Bolivia. Se trata de un estudio mixto con alcance causal que mediante un pre experimento con modelo pretest-postest aplicado a 65 individuos pudo evaluar la preferencia de marca de 3 cervezas, asimismo mediante la observación se comparó las estrategias de marketing aplicadas por estas marcas. Como resultado se comprobó que no existe relación entre estándares de calidad de cerveza (aroma, color, sabor espuma y cuerpo) y preferencia de marca, sino es el neuromarketing el que determina el comportamiento del consumidor. Se concluye que se trata de un comportamiento irracional e inconsciente, motivado por emociones, que debe ser enfocado desde el neuromarketing.
\end{abstract}

Palabras clave: Neuromarketing; comportamiento; compras; psicología; consumidores, cerveza

\begin{abstract}
The application of neuromarketing has revolutionized the study of consumer behavior, generating benefits for various sectors, including the beer industry. The objective of this study was to determine the most relevant influencing factor in the purchasing behavior of beer consumers in Sucre, Bolivia. It is a mixed study with a causal scope that by means of a preexperiment with a pre-test-post-test model applied to 65 individuals, it was able to evaluate the brand preference of 3 beers, also by means of observation, and the marketing strategies applied by these brands were compared. As a result, it was found that there is no relationship between beer quality standards (aroma, color, taste, foam and body) and brand preference, but it is neuromarketing that determines consumer behavior. It is concluded that it is an irrational and unconscious behavior, motivated by emotions, which must be approached from neuromarketing.
\end{abstract}

Key words: Neuromarketing; behaviour; purchases; psychology, consumers, Beer
Segovia Jaramillo Vladimir vladimirsegoviajaramillo.vsj@gmail.com Orcid: 0000-0002-0663-6929

Universidad Andina Simón Bolívar, Bolivia

Licenciatura en Administración de Empresas e Ingeniería Comercial (U.S.F.X.CH.). Maestría en Administración de Empresas MBA (U.A.S.B.). Jefe y Responsable de control de Inventarios - CIS S.R.L. (2 años), Gerente de Marketing INNOVA S.R.L. (3 años), Jefe de Marketing -GAMBABOL S.R.L. (2 años), otros. Amplia experiencia en la industria alimenticia. Universidad Andina Simón Bolívar, Bolivia. 


\section{Resumo}

A aplicação do neuromarketing revolucionou o estudo do comportamento do consumidor, gerando benefícios para diversos setores, inclusive para a indústria cervejeira. $\mathrm{O}$ objetivo deste estudo foi determinar o fator de influência mais relevante no comportamento de compra dos consumidores de cerveja em Sucre, Bolívia. É um estudo misto de âmbito causal que, através de um pré-experimento com um modelo pré-teste-pós-teste aplicado a 65 indivíduos, foi capaz de avaliar a preferência de marca em 3 cervejas, também por meio de observação, as estratégias de marketing aplicadas por essas marcas foram comparadas. Como resultado, constatou-se que não existe relação entre os padrões de qualidade da cerveja (aroma, cor, sabor, espuma e corpo) e a preferência pela marca, mas é o neuromarketing que determina o comportamento do consumidor. Conclui-se que é um comportamento irracional e inconsciente, motivado por emoções, que deve ser abordado a partir do neuromarketing.

Palavras-chave: Neuromarketing; comportamento; compras; psicologia; consumidores de cerveja

\section{INTRODUCCIÓN}

En la industria de la cerveza cada vez emergen nuevas tendencias de consumo, a las cuales las empresas se van acomodando para garantizar su crecimiento. Una de las principales tendencias en los últimos años ha sido la diversificación en sabores y especialidades, debido a que la preferencia de los consumidores está centrada en la innovación (Ablin, 2014).

Así, está claro que las nuevas generaciones de consumo han cambiado sus viejos hábitos y están reconfigurando la forma en cómo compran y se relacionan con las marcas. El comprador de hoy busca experiencias nuevas, es decir, ya no se enfoca únicamente en el sabor de la cerveza, sino en un sin número de aspectos que en la mayoría de los casos escapa de su conocimiento. Por esto, el reto para tomadores de decisiones y publicistas está en conocer cómo su cliente potencial siente emociones (Álvarez del Blanco, 2010).

Los cambios actuales en el marketing están enfocados en la aplicación de las neurociencias, específicamente en el neuromarketing. En este sentido, se afirma que las personas toman decisiones emocionalmente y de manera inconsciente, dicho en porcentajes, según Kumar y Singh (2015), el 85\% de las decisiones están basadas en la emoción y sólo después de haber tomado esa decisión desde la emoción se busca una justificación racional, según Kahneman (2013) es el $88 \%$.

Para estudiar el mercado de cerveza a profundidad, se tomó como objeto de estudio la ciudad de Sucre, Bolivia. Este mercado en los últimos años ha experimentado incremento de la competitividad y la rivalidad de competidores, debido al fortalecimiento de empresas internacionales, lo cual exige a las empresas locales la introducción de nuevas técnicas y herramientas de vanguardia que consigan descubrir o activar la necesidad del consumidor. Cabe mencionar que el mercado está liderado por 4 cervezas: Paceña, Huari, Sureña y Potosina, asimismo, el tipo de cerveza que más se produce y demanda es "lager de cebada".

La Cervecería Boliviana Nacional (CBN), es una empresa internacional emblemática en Bolivia, cubre más del $80 \%$ del mercado nacional, con plantas cerveceras en La Paz, Oruro, Cochabamba, Santa Cruz y Tarija, entre sus marcas, Paceña y Huari son líderes en la ciudad de Sucre. Cabe resaltar que la CBN está enfocada hacia las nuevas tendencias, tanto en la diversificación de sabores, como en el marketing. En paralelo se tiene a Sureña, ésta es una cervecería local que tiene 
reconocimiento de marca en Sucre, está enfocada en una estrategia de diferenciación y ofrece al consumidor un producto con altos estándares de calidad, no obstante, el consumidor no percibe a la marca Sureña como la mejor, en consecuencia, Paceña y Huari tienen mayor cuota de mercado en esta ciudad. Por su parte, la cervecería Potosina está orientada al liderazgo en costos y tiene una cuota de mercado reducida, inferior a Sureña, (Sandoval, 2019).

En la industria cervecera local se observa descuido en la aplicación de técnicas de marketing, aspecto que se refleja en empresas como Sureña y Potosina, lo cual repercute directamente en sus ventas. Estas empresas enfocan sus esfuerzos en mejorar características propias del producto, como su sabor, creyendo que este es el principal factor que consideran los consumidores al momento de comprar una marca de cerveza. Sin embargo, no existe prueba científica para afirmar que el consumidor de cerveza local toma decisiones de compra racionales, por el contrario, existen estudios a nivel internacional que afirman que el consumo de cerveza está altamente influenciado por decisiones emocionales e inconscientes, (Braidot, 2006).

En este sentido es importante determinar las necesidades de los consumidores en la industria cervecera desde una perspectiva diferente a la que se ha estado manejando en Bolivia, es por eso que esta investigación determinar cuál es el factor de influencia más relevante en el comportamiento de compra de los consumidores de cerveza en la ciudad de Sucre, con este fin, se parte del postulado de que la aplicación de técnicas de neuromarketing es determinante en la decisión de compra de los consumidores.
Entonces, estando al tanto de las necesidades existentes en la industria cervecera local, como el crecimiento de la competencia y el desconocimiento de las necesidades de los consumidores, surge la imperiosa necesidad de contar con este estudio, el cual aporta directrices enfocadas a entender el comportamiento de compra del consumidor de este producto. Dicho de otra forma, en la medida que las empresas locales puedan determinar con mayor claridad los atributos de la cerveza más relevantes para los consumidores, podrán elevar su eficiencia y se enfocarán en el desarrollo de estrategias efectivas que les permitan incrementar su participación en el mercado.

A continuación, se presentan bases teóricas que respaldan el estudio, referidas a: neuromarketing y diseños pre-experimentales.

\section{Neuromarketing}

Las principales definiciones del neuromarketing: a) aplicación de la neurociencia al marketing mediante la imaginería del cerebro, el escaneo y otras actividades cerebrales registrables con equipos tecnológicos, de tal modo que la información que procesan puede ser más reveladora que las encuestas tradicionales (Doley, 2015), b) modelo de marketing que permite visualizar y está ligado a la neurociencia, en este sentido el neuromarketing aplicado usa la neurociencia para mejorar ventas (Renvoise, y Moryn, 2007). c) cualquier esfuerzo de marketing que use los métodos de la ciencia del cerebro, es decir, es solucionar los mismos problemas que todos los tipos de investigación de mercado quieren resolver, pero mejor (Genco; Pohlmann, y Steidl, 2013), d) estudia los procesos cerebrales que revelan la conducta y 
la toma de decisiones de las personas, en los campos de acción del marketing tradicional: inteligencia de mercado, diseño de productos y servicios, comunicaciones, precios, branding, posicionamiento, targeting, canales y ventas, (Braidot, 2009), e) es recoger información del medio ambiente, analizarla y tomar decisiones (Montterlini, 2008).

Según Monge y Fernández (2011), existen tres ideas principales que son las que ayudan a entender esta modificación en la idea preconcebida de consumidor: a) las decisiones que toman las personas son irracionales, b) para entender al consumidor hay que entender cómo piensan, c) los consumidores toman decisiones con base a información que poseen, pero de la cual no son conscientes. Así, la noción de estímulo está vinculada, como revela Millward, (2009), a incitar una actividad, operación o función.

En este sentido, Millward (2009) dice que las actitudes pueden impulsar las decisiones de compra. En este sentido González y Triana (2018) dice que las actitudes constan de tres componentes: 1) afectivo, formado por los sentimientos o emociones de una persona por el objeto, tema o ideal. 2) cognitivo, las imágenes mentales, comprensión e interpretaciones de la persona, objeto o tema. 3) conativo, las intenciones, acciones o comportamiento del individuo.

Para la aplicación efectiva del neuromarketing es importante conocer el proceso de respuesta del consumidor que induce a un comportamiento específico como la compra de un producto y la influencia de las actividades promocionales de la organización en las respuestas del consumidor, (Álvarez del Blanco, 2010).

Por su parte, Torres (2012), se enfoca en el neuromarketing para comprender a los clientes y crear fidelización. Un ejemplo clásico de su aplicación relacionada a la fidelización es el Pepsi Test, de 1970, ejemplos clásicos en el que se realizaron numerosas pruebas ciegas de Cocacola y Pepsi, revelándose que Pepsi era la preferida en términos de sabor, sin embargo, cuando los consumidores veían las marcas escogían Coca-Cola, la mayoría de las personas declararon preferir el sabor de Coca-Cola, aunque no pudieron explicar por qué (De Andreis, 2012).

En la actualidad existe un sin número de aplicaciones y experimentos que buscan conocer la mente del consumidor $y$, surge entonces la preocupación de debatir sobre ética, en este tenor, para León (2010), es importante analizar el alcance que tiene esta aplicación de la neurociencia al marketing, cuestiona y propone soluciones frente a la ética y la moral que deben limitar y guiar el buen uso de esta maravillosa herramienta.

\section{Diseños pre-experimentales}

Los diseños pre-experimentales son denominados comúnmente experimentos crudos, no emplean ningún procedimiento aleatorio para controlar los efectos causados por las variables extrañas. Dentro de este grupo están los pretest-postest con un grupo, que son un tipo de diseño aplicado en el que un grupo de unidades prueba se mide dos veces, es decir, se realizan dos mediciones, una previa al tratamiento $\mathrm{X}$, simbolizada por $\mathrm{O}_{1} \mathrm{Y}$ otra posterior $\mathrm{O}_{2}$, permitiendo determinar el efecto del tratamiento comparando $\mathrm{O}_{2}-\mathrm{O}_{1}$. Se sabe que en este tipo de experimentos no hay control de variables extrañas (Manterola, y Otzen 2015). 
MÉTODO

El estudio se realizó bajo el enfoque mixto con alcance causal, el análisis de la información se usó la técnica cualitativa. El aporte cuantitativo se desarrolló gracias a la aplicación de un pre-experimento que permitió demostrar la relación entre las variables que determinan el proceso de compra de cerveza. Las técnicas empleadas para la recolección de los datos fueron la observación y la entrevista.

La técnica de la observación se implementó para determinar el comportamiento del consumidor de cerveza en la sociedad, conforme sucede en el entorno, respecto a su toma de decisiones. Permitió visibilizar los factores externos (posicionamiento de marca, marketing $y$ publicidad adheridas al neuromarketing) e internos (emociones, gustos e intereses) que afectan la decisión de compra y su preferencia por una determinada marca. La información fue recopilada mediante instrumento de registro

Asimismo, mediante la observación se pudo determinar las tendencias actuales de consumo que se están dando en el medio y que afectan el proceso comercial de la industria cervecera en la ciudad de Sucre. Para la entrevista se usó un cuestionario dirigidos a gerentes de las principales cervecerías del medio y profesionales que facilitaron la recogida de datos.

Se analizó comparativamente tres marcas de cerveza: Paceña, Huari y Sureña Bicentenario. Para determinar los individuos a participar en el experimento y conformar los grupos de estudio, se tomó en cuenta dos variables demográficas: género y edad. Con un total de 65 personas sometidas al estudio, clasificadas en tres grupos heterogeneos según edad: 1) 35 personas entre 18 y 28 años, 2) 15 individuos entre 29 y 39 años y, c) 15 personas mayores a 39 años. En todos los grupos el $70 \%$ de los participantes fueron varones y $30 \%$ mujeres. El experimento realizado pertenecía a la categoría de diseño pre-experimental con modelo de pretest-postest.

Se obtuvo control moderado de dos variables extrañas al experimento: 1) Historia $(H)$, se pudo controlar esta variable extraña escogiendo ciertas condiciones para realizar el experimento: un ambiente abierto, temperatura ambiente de 28 grados, cielo despejado, baja o nula contaminación ambiental (eliminación de ruido y olores que distorsionen la degustación). Asimismo, las tres marcas de cervezas se encontraban en una temperatura ideal para el consumo $\left(5^{\circ} \mathrm{C}\right) .2$ ) Madurez (MA), se controló esta variable ya que el experimento no fue de larga duración y fue planteado de manera que resultase entretenida para los participantes, es así como no presentaron cansancio ni aburrimiento.

\section{Simbología}

- X: Degustación de la cerveza a ciegas

- $\mathrm{O}_{1}$ : Medición de la unidad de prueba previa a la degustación a ciegas.

- $\mathrm{O}_{2}$ : Medición de la unidad de prueba posterior a la degustación a ciegas.

\section{Proceso del pre-experimento}

El pre-experimento se realizó en ambiente controlado, el cual consistió en hacer la degustación a ciegas de tres diferentes marcas de cerveza de una misma clase que es lager de cebada: Huari, Paceña y Sureña Bicentenario. Siguiendo el procedimiento: a) Se procedió al llenado de una ficha, en donde la unidad de prueba seleccionó la cerveza que consume preferentemente $y / o$ la de mayor agrado y 
posteriormente describió algunas características acerca de los motivos por los cuales prefiere una marca. b) La unidad de prueba se sometió a la degustación a ciegas, el investigador proporcionó tres vasos con cerveza Huari, Paceña y Sureña Bicentenario (sin identificación de marca). c) Una vez realizada la degustación, los consumidores evaluaron algunas características de la cerveza, posteriormente escogieron la que más les agradó y reconocieron las marcas. d) Se contrastaron los resultados del Pretest con los de la degustación a ciegas. e) Finalmente se formularon conclusiones.

\section{Metodología de evaluación}

Durante el proceso se definieron estándares de calidad a evaluar adecuados para el tipo de cerveza que se toma en cuenta para el experimento (lager de cebada), para lo cual se utilizó una escala Likert que permitió determinar el nivel de calidad que tiene cada cerveza para los distintos grupos de consumidores y para cada consumidor en particular. Estos estándares de calidad a evaluar fueron traducidos a ítems: a) aroma, el ideal de este tipo de cerveza es tener gran parecido al de la cebada y que no existan otros olores que lo distorsionen b) color, dorado intenso, c) sabor, a cebada, la cerveza no debe ser ni muy amarga ni muy acida, se debe encontrar un punto medio, d) espuma, consistencia y/o permanencia que la espuma debe tener una vez servida en el vaso, el tiempo ideal es aproximadamente 30 segundos, e) cuerpo, conocido también como retrogusto y está referido a la permanencia del sabor de la cerveza en la lengua.

\section{RESULTADOS}

En este apartado se presentan los resultados del pre-experimento con modelo pretest-postest aplicado a 65 individuos, hombres y mujeres, mayores de edad clasificados en 3 grupos: a) grupo 1, de 18 a 28 años, b) grupo 2, de 29 a 39 años $y$, c) mayores a 39 años. La tabla 1 muestra la preferencia de consumo en el test previo a la degustación.

Tabla 1. Preferencia de consumo en el test previo a la degustación

\begin{tabular}{lcccccc}
\hline & \multicolumn{2}{c}{ Grupo 1 } & \multicolumn{2}{c}{ GRUPOS DE ESTUDIO } & \multicolumn{2}{c}{ Grupo 3 } \\
\cline { 2 - 8 } \multicolumn{1}{c}{ MARCAS } & $\mathbf{N}^{\circ} \mathbf{P .}$ & $\%$ & $\mathbf{N}^{\circ} \mathbf{P .}$ & $\%$ & $\mathbf{N}^{\circ}$ P. & $\%$ \\
\hline Huari & 16 & $46 \%$ & 7 & $47 \%$ & 9 & $60 \%$ \\
Paceña & 19 & $54 \%$ & 8 & $53 \%$ & 6 & $40 \%$ \\
Sureña B. & 0 & $0 \%$ & 0 & $0 \%$ & 0 & $0 \%$ \\
\hline Total & 35 & $100 \%$ & 15 & $100 \%$ & 15 & $100 \%$ \\
\hline
\end{tabular}

Según se observa en la tabla 1 , del total de las personas que conforman el grupo 1 en el test previo, el $54 \%$ mencionaron que la marca de cerveza de su preferencia es Paceña, seguida de Huari con un $46 \%$. De manera similar en el grupo 2, la cerveza preferida fue Paceña con un 53\%, superando a Huari que alcanzó una preferencia del $47 \%$. En el tercer grupo, la cerveza preferida es Huari con un $60 \%$, seguida de Paceña con un $40 \%$. 
Destaca la preferencia por Huari y Paceña en los tres grupos de estudio. Asimismo, los individuos mencionaron que su preferencia hacia las cervezas Huari y Paceña está dada principalmente por su calidad y sabor. Además, argumentaron que la marca Sureña en general no tiene la misma calidad que las otras. Resalta que la cerveza Sureña Bicentenario tiene una preferencia del $0 \%$ en los tres grupos de estudio, lo que significa que nadie escogió esta marca de cerveza como su preferida.

En relación con la preferencia según género, la cerveza preferida por los hombres del grupo 1 fue Paceña con un 58\%, seguida de Huari con un $42 \%$, en el segmento femenino se puede observar un resultado distinto ya que la marca con mayor preferencia es Huari con un $55 \%$, seguida de Paceña con un $45 \%$. Dentro del segundo grupo de estudio se observa que en el segmento masculino existe una preferencia del $60 \%$ por Paceña, seguida de Huari con un $40 \%$, no obstante, sucede lo contrario en el segmento femenino, ya que la preferencia está dada por Huari con un $60 \%$, seguida de Paceña con un $40 \%$. En el tercer grupo de estudio se pudo observar que la preferencia en el segmento masculino es compartida por Paceña y Huari con un $50 \%$ para cada una, sin embargo, no sucede lo mismo en el segmento femenino, ya que existe una marcada preferencia hacia Huari $(80 \%$ escogió Huari y $20 \%$ Paceña).

En cuanto a atributos de consumo valorados en la cerveza, en el pretest se pidió escoger solamente el principal atributo, así, el 100\% del grupo 1 escogió el sabor. Por su parte, el segundo grupo de edad afirma que el sabor es el principal atributo con un $93 \%$, seguido del cuerpo con un $7 \%$, en el tercer grupo de estudio el $80 \%$ define el sabor como principal atributo y $20 \%$ el cuerpo.

A manera de resumen se puede mencionar que el principal atributo por el cual los consumidores guían su preferencia de consumo de cerveza es el sabor.

Tabla 2. Preferencia de consumo en la degustación a ciegas

\begin{tabular}{|c|c|c|c|c|c|c|}
\hline \multirow{3}{*}{ MARCAS } & \multicolumn{6}{|c|}{ GRUPOS DE ESTUDIO } \\
\hline & \multicolumn{2}{|c|}{ Grupo 1} & \multicolumn{2}{|c|}{ Grupo 2} & \multicolumn{2}{|c|}{ Grupo 3} \\
\hline & $\mathbf{N}^{\circ} \mathbf{P}$. & $\%$ & $\mathbf{N}^{\circ} \mathbf{P}$. & $\%$ & $\mathbf{N}^{\circ} \mathbf{P}$. & $\%$ \\
\hline Huari & 13 & $37 \%$ & 5 & $33 \%$ & 3 & $20 \%$ \\
\hline Paceña & 5 & $14 \%$ & 4 & $27 \%$ & 6 & $40 \%$ \\
\hline Sureña B. & 17 & $49 \%$ & 6 & $40 \%$ & 6 & $40 \%$ \\
\hline Total & 35 & $100 \%$ & 15 & $100 \%$ & 15 & $100 \%$ \\
\hline
\end{tabular}

Según se presentó en la tabla 2 , en la degustación a ciegas $49 \%$ de los individuos del primer grupo mencionaron que la marca de cerveza que más les agradó y por ende de su preferencia fue Sureña Bicentenario, seguida de Huari con un $37 \%$ y por último Paceña con tan solo un $14 \%$. En el segundo grupo de estudio pasó algo similar, la cerveza de mayor preferencia fue Sureña Bicentenario con un $40 \%$, seguida de Huari 
con un $33 \%$ y en último lugar Paceña con un $27 \%$. Dentro del tercer grupo se manifestó una igualdad respecto a la preferencia de la cerveza, ya que tanto Huari como Sureña Bicentenario alcanzaron un $40 \%$, en tanto que Paceña solo alcanzó a tener un $20 \%$.

Referente a la preferencia de consumo según género, para el segmento masculino del grupo 1 existe una preferencia del 50\% por la cerveza Sureña Bicentenario, seguida de Huari con un $33 \%$ y por último Paceña con un $17 \%$, de igual forma en el segmento femenino Sureña bicentenario y Huari son las de mayor agrado con un $45 \%$ y por último Paceña con tan solo un $10 \%$. En la degustación del segundo grupo se pudo observar que para el segmento masculino la cerveza preferida fue Sureña Bicentenario con un $50 \%$, posteriormente se encuentra Huari con un $40 \%$ y por último Paceña con $10 \%$, para el segmento femenino se observa una marcada preferencia por Paceña con un $60 \%$ seguida por Huari y Sureña Bicentenario con un $20 \%$ para cada una. Dentro del tercer grupo se observa que el segmento masculino tuvo una leve preferencia por Sureña Bicentenario con un $40 \%$, y por debajo de ella se encuentran Paceña y Huari con un $30 \%$ cada una, finalmente, el segmento femenino se inclinó en primer lugar por Paceña con un 60\%, seguida de Sureña Bicentenario con un $40 \%$.

En relación con el motivo de elección de la cerveza de su preferencia, en el grupo 1 el 94\% manifestó que el principal factor para que escojan su cerveza preferida fue el sabor, seguida del cuerpo con un $6 \%$. En el grupo 2 el $80 \%$ manifestó que el principal factor para que escojan su marca preferida fue el sabor y el $20 \%$ indicó que el cuerpo fue lo que más les llamó la atención. De igual forma que en los anteriores grupos de estudio el sabor predomina en los consumidores del tercer grupo, con un $60 \%$ y el cuerpo de la cerveza fue considerado el principal motivo para el $40 \%$.

Seguidamente, se midió los atributos que cada sujeto asignó a cada una de las tres cervezas (a ciegas) en escala Likert (véase gráfico 1).

\section{Grupo 1}

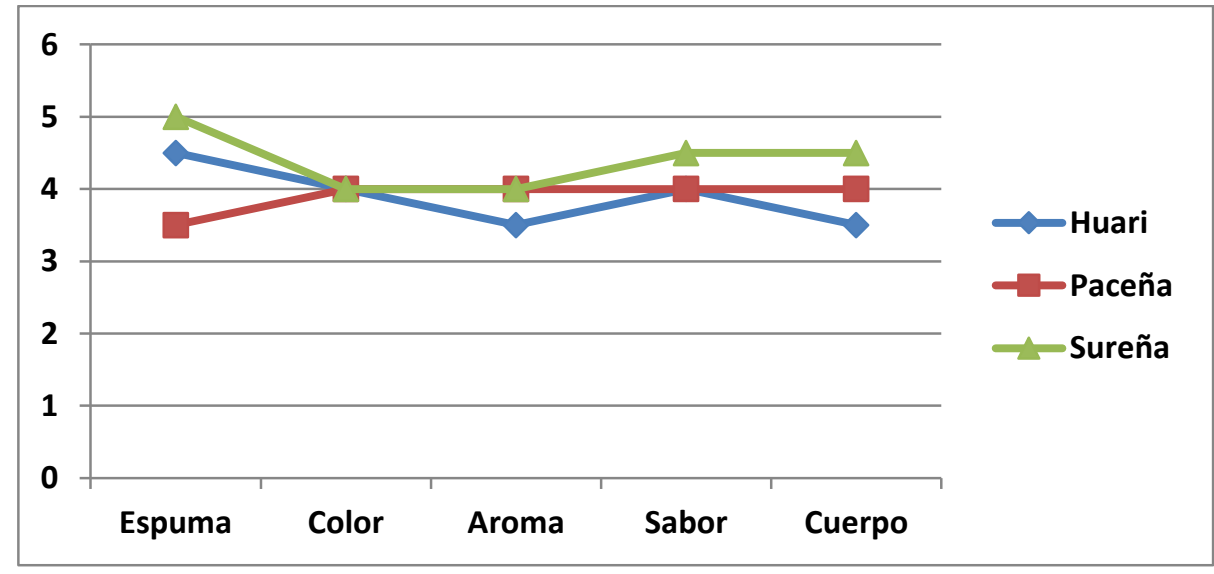




\section{Grupo 2}

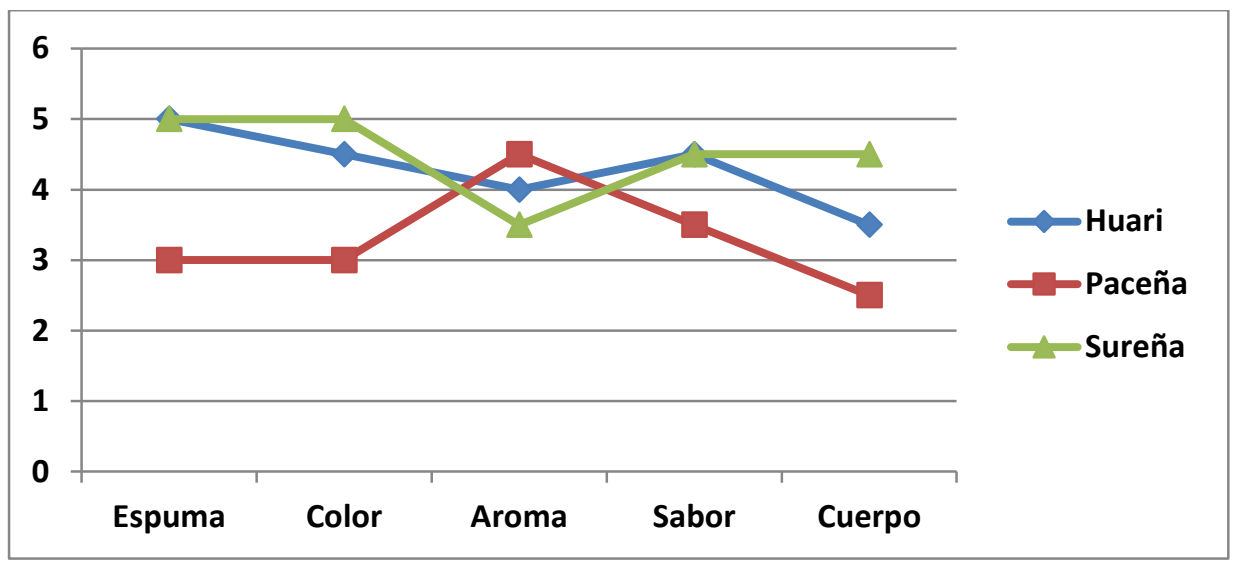

Grupo 3

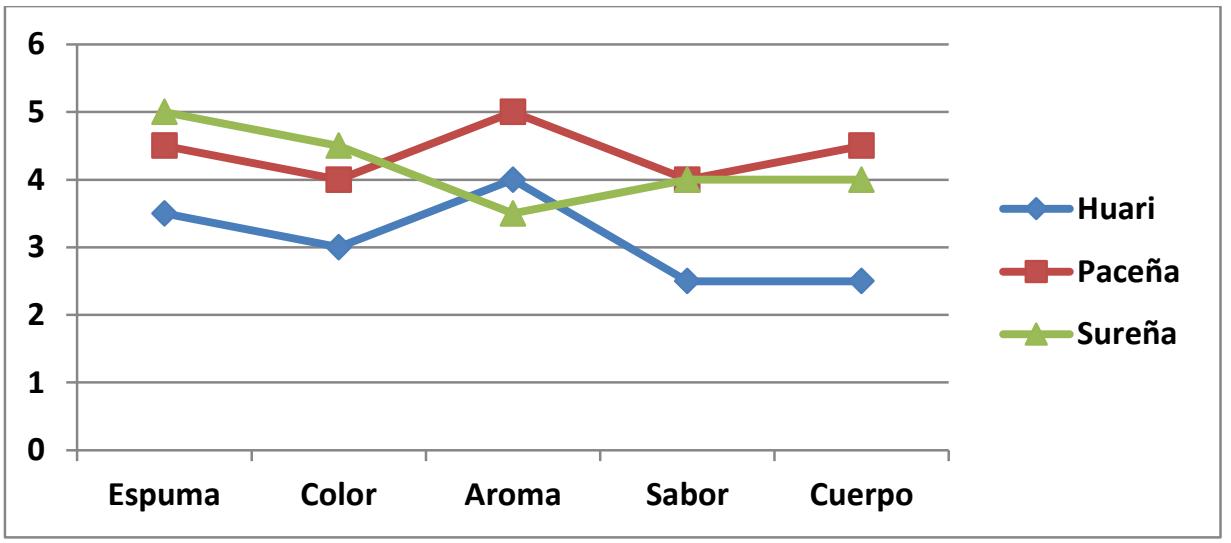

Gráfico 1. Calificación promedio de los atributos (Huari - Paceña - Sureña Bicentenario).

Según se aprecia en el gráfico 1 en una escala del 1 al 5 (siendo 1 pésimo y 5 excelente), la percepción después de la degustación a ciegas del primer grupo fue: Sureña Bicentenario obtuvo mayor calificación en sabor, cuerpo y espuma. Paceña en general obtuvo una calificación de 4 para todos sus atributos, a excepción de espuma, atributo en el cual obtuvo un puntaje de 3,5. Huari estuvo por debajo de ambas marcas en dos atributos, en el aroma y el cuerpo; sin embargo, al margen de aquello obtuvo una buena calificación en espuma.
En el grupo 2, la cerveza Sureña Bicentenario obtuvo una mayor calificación entre las tres marcas analizadas principalmente en cuatro de sus atributos: sabor, cuerpo, espuma y color, en los cuales tiene la máxima calificación. Sin embargo, tiene una muy baja calificación en cuanto se refiere al aroma. La cerveza Huari presenta una puntuación alta en casi todos sus atributos a excepción del cuerpo en el que tiene una puntuación de 3.5. Paceña es la cerveza que obtuvo la más baja calificación en todos los atributos analizados. 
Continuando con el grupo 3, la cerveza Paceña obtuvo una mayor puntuación en la mayoría de los atributos, principalmente en aroma en la cual tiene una puntuación de 5 , espuma y cuerpo fueron calificados con 4.5. Respecto a Sureña Bicentenario, se puede observar que mantiene una puntuación alta en espuma y color, pero la puntuación en aroma es baja. La cerveza Huari tuvo la menor puntuación en todos sus atributos a excepción del aroma en el cual tiene un puntaje de cuatro.
Sintetizando, en promedio Sureña tiene mayor valoración, seguida de Huari y en último Paceña, en los grupos 1 y 2 . Sin embargo, en el grupo 3 la cerveza que obtuvo una mayor puntuación fue Paceña, seguida de Sureña Bicentenario y Huari.

Gracias a la prueba a ciegas se pudo determinar la capacidad de identificación de la cerveza y el porcentaje de confusión con otras marcas (tabla 3 ).

Tabla 3. Identificación de la cerveza / confusión con otras marcas

\begin{tabular}{lccccccccc}
\hline & \multicolumn{3}{c}{ Grupo 1 } & \multicolumn{9}{c}{ Grupos de Estudio } \\
Grupo 2 & \multicolumn{3}{c}{ Grupo 3 } \\
MARCAS & Huari & Sureña & Paceña & Huari & Sureña & Paceña & Huari & Sureña & Paceña \\
\hline Huari & $30 \%$ & $35 \%$ & $45 \%$ & $20 \%$ & $33 \%$ & $47 \%$ & $20 \%$ & $47 \%$ & $33 \%$ \\
Sureña & $30 \%$ & $35 \%$ & $40 \%$ & $47 \%$ & $27 \%$ & $40 \%$ & $33 \%$ & $27 \%$ & $40 \%$ \\
Paceña & $40 \%$ & $30 \%$ & $15 \%$ & $33 \%$ & $40 \%$ & $13 \%$ & $47 \%$ & $26 \%$ & $27 \%$ \\
\hline Total & $\mathbf{1 0 0 \%}$ & $\mathbf{1 0 0} \%$ & $\mathbf{1 0 0 \%}$ & $\mathbf{1 0 0 \%}$ & $\mathbf{1 0 0 \%}$ & $\mathbf{1 0 0 \%}$ & $\mathbf{1 0 0 \%}$ & $\mathbf{1 0 0 \%}$ & $\mathbf{1 0 0 \%}$ \\
\hline
\end{tabular}

Según se puede apreciar en la tabla 3 , se analizó el porcentaje de aciertos respecto a la preferencia consciente y a la preferencia en la cata a ciegas, los resultados se resumen a continuación.

En el primer grupo la confusión de la cerveza Huari con Sureña Bicentenario fue un $40 \%$ y con Paceña fue un $30 \%$, hubo sólo un $30 \%$ de aciertos. Una situación similar se dio con Sureña Bicentenario, confundiéndose el $35 \%$ con Huari y el $30 \%$ con Paceña, acertando un $35 \%$ de las personas. Respecto a Paceña, hubo una confusión con Huari del $45 \%$ y con Sureña Bicentenario del 40\%, acertando únicamente un $15 \%$.

Por su parte, en el segundo grupo existió confusión de la cerveza Huari con Sureña Bicentenario en un $47 \%$ y con Paceña en un $20 \%$; habiendo acertado solo el $33 \%$. Una situación similar se dio con Sureña
Bicentenario, confundiéndose el $33 \%$ con Huari y el $40 \%$ con Paceña, acertando un $27 \%$ de las personas. Respecto a la cerveza paceña hubo una confusión con Huari del $47 \%$ y con Sureña Bicentenario del $40 \%$, acertando únicamente un $13 \%$.

Para terminar, en el tercer grupo se confundió la cerveza Huari con Paceña en un $47 \%$ y con Sureña Bicentenario en un 33\%; habiendo acertado solo el $20 \%$. En el caso de la cerveza Paceña el $40 \%$ la confundió con Sureña Bicentenario y un $33 \%$ con Huari, acertando un $33 \%$ de las personas. Respecto a la cerveza Sureña Bicentenario hubo una confusión con Huari del $47 \%$ y con Sureña Bicentenario del $27 \%$, acertando únicamente un $26 \%$.

La tabla 4 presenta una relación entre los resultados obtenidos en el test previo y la degustación a ciegas. 
Tabla 4. Test previo Vs degustación a ciegas

\begin{tabular}{|c|c|c|c|c|c|c|c|c|c|c|c|c|}
\hline \multirow{3}{*}{ MARCAS } & \multicolumn{6}{|c|}{$\begin{array}{c}\text { TEST PREVIO } \\
\text { Grupos de Estudio }\end{array}$} & \multicolumn{6}{|c|}{$\begin{array}{c}\text { DEGUSTACIÓN A CIEGAS } \\
\text { Grupos de Estudio }\end{array}$} \\
\hline & \multicolumn{2}{|c|}{ Grupo 1} & \multicolumn{2}{|c|}{ Grupo 2} & \multicolumn{2}{|c|}{ Grupo 3} & \multicolumn{2}{|c|}{ Grupo 1} & \multicolumn{2}{|c|}{ Grupo 2} & \multicolumn{2}{|c|}{ Grupo 3} \\
\hline & $\mathbf{N}^{\circ} \mathbf{P}$. & $\%$ & $\mathbf{N}^{\circ} \mathbf{P}$. & $\%$ & $\mathbf{N}^{\circ} \mathbf{P}$. & $\%$ & $\mathbf{N}^{\circ} \mathbf{P}$. & $\%$ & $\mathbf{N}^{\circ} \mathbf{P}$. & $\%$ & $\mathbf{N}^{\circ} \mathbf{P}$. & $\%$ \\
\hline Huari & 16 & $46 \%$ & 7 & $47 \%$ & 9 & $60 \%$ & 13 & $37 \%$ & 5 & $33 \%$ & 3 & $20 \%$ \\
\hline Paceña & 19 & $54 \%$ & 8 & $53 \%$ & 6 & $40 \%$ & 5 & $14 \%$ & 4 & $27 \%$ & 6 & $40 \%$ \\
\hline Sureña & 0 & $0 \%$ & 0 & $0 \%$ & 0 & $0 \%$ & 17 & $49 \%$ & 6 & $40 \%$ & 6 & $40 \%$ \\
\hline Total & 35 & $100 \%$ & 15 & $100 \%$ & 15 & $100 \%$ & 35 & $100 \%$ & 15 & $100 \%$ & 15 & $100 \%$ \\
\hline
\end{tabular}

Según se vio en la tabla 4, al contrastar los resultados del pre-test con la degustación a ciegas se encontró información valiosa. En el primer grupo, las personas conscientemente manifestaron su preferencia por Paceña 54\% y Huari $46 \%$. Sin embargo, en la prueba a ciegas la cerveza preferida fue Sureña Bicentenario (49\%), seguida de Huari con un $37 \%$ y Paceña alcanzado tan solo un $14 \%$. En el grupo 2, las personas conscientemente dijeron tener preferencia por Paceña y Huari, sin embargo, al contrastar los resultados con los de la degustación a ciegas se observa incongruencia ya que Sureña Bicentenario es la cerveza de preferencia con un $40 \%$ de los individuos. De manera similar el grupo 3, en el test previo expresó una clara preferencia por Huari con un $60 \%$ y Paceña con un $40 \%$, contrariamente en la degustación a ciegas Sureña Bicentenario y Paceña fueron escogidas por $40 \%$ de los individuos cada una y sólo $20 \%$ dijo que le gustó más la cerveza Huari.

Se evidencia que la decisión de compra se ve fuertemente influenciada por variables externas al producto en sí, variables a las que el consumidor ha estado expuestas consciente e inconscientemente (posicionamiento de marca, publicidad, empaque, precio, referencias). En la prueba previa Paceña y Huari tienen una marcada preferencia sobre Sureña Bicentenario, no obstante, en la segunda etapa, cuando los sujetos de estudio evalúan el producto sin conocer la marca, se ven obligados a considerar los estándares de calidad del producto (aroma, color, sabor espuma y cuerpo), se manifiesta entonces preferencia por la cerveza Sureña Bicentenario. Esta cerveza tiene el mejor sabor y calidad respecto de sus competidores, motivo por el cual es la marca de mayor preferencia en la degustación a ciegas; Sin embargo, esta estadística no se manifiesta en el consumo de cerveza de las personas en la ciudad de Sucre, esto debido a que el sabor y la calidad no son determinantes en el proceso de compra de cerveza.

Para sintetizar, existe diferenciación según género del consumidor, el segmento masculino prefiere comprar cerveza Paceña, a diferencia del segmento femenino que valora mejor la cerveza Huari. Ambos géneros atribuyen al sabor como principal determinante de la compra. Sin embargo, en la prueba a ciegas la cerveza Huari pierde presencia en el segmento femenino, principalmente en el grupo de consumidores de 40 años o más y, la cerveza Sureña Bicentenario gana liderazgo entre el consumidor femenino. En decir, las mujeres dicen que prefieren Huari por su sabor, en contraste, al evaluar el sabor y no la marca escogen Sureña Bicentenario. Algo similar sucede en el segmento masculino, ellos dicen 
que prefieren Paceña principalmente por su sabor, no obstante, al desconocer la marca escogen Sureña Bicentenario.

El componente cualitativo de la investigación permitió observar la estrategia de marketing que aplica cada una de las marcas, como resultado se determinó que Sureña Bicentenario aplica publicidad tradicional, asimismo su propuesta de valor está enfocada en resaltar los atributos del producto. Por el contrario, Paceña y Huari aplican neuromarketing, apuestan por generar emociones en sus clientes y lo hacen a través de una serie de estudios que se traducen en acciones estratégicas, entre ellas la publicidad adherida el neuromarketing. En cuanto a la estrategia de Sureña Bicentenario, se encontró que la marca se dirige al cerebro racional de los consumidores, promoviendo la compra a través de resaltar atributos como calidad y procedencia de la empresa (con el slogan "consume lo nuestro").

\section{CONCLUSIONES}

La metodología empleada, que combina métodos cuantitativos (pre-experimento con modelo de pretest-postest) y cualitativos (entrevistas y observación), permitió alcanzar el objetivo propuesto determinando la variable más relevante en el comportamiento de compra de los consumidores de cerveza en la ciudad de Sucre. Se comprobó que no existe relación entre los estándares de calidad (aroma, color, sabor espuma y cuerpo) y la preferencia de marca de cerveza, ya que se no se encontró correlación entre la preferencia de cerveza en el pretest y en el postest. Por tanto, se infiere que la preferencia de marca no está determinada por los atributos de la cerveza, sino más bien por variables externas al producto (marketing y publicidad). Asimismo, se puede puntualizar y determinar que el principal determinante del comportamiento de compra de cerveza en Sucre, es la publicidad adherida al neuromarketing, que al despertar emociones mueve al consumidor a comprar una marca independientemente de su sabor y calidad. Lo interesante es que el consumidor no es consciente de este hecho, ya que la publicidad adherida al neuromarketing va directamente al subconsciente.

Teniendo en cuenta que el comportamiento de compra de los consumidores de cerveza no es un proceso racional, sino más bien emocional del cual no tienen conciencia, el éxito de las empresas cerveceras en la ciudad de Sucre depende en gran medida de aplicar publicidad adherida al neuromarketing que vaya directamente al subconsciente de los consumidores. Por tanto, las empresas de cerveza locales deben cambiar su enfoque publicitario, dejando de lado los antiguos paradigmas acerca del comportamiento del consumidor, entendiendo que no se trata de un consumidor racional. En este caso, aplicar una estrategia afectiva permitirá elevar el posicionamiento de marca en la mente del cliente.

\section{REFERENCIAS BIBLIOGRÁFICAS}

Ablin, A. (2014). El mercado de la cerveza. Informe sectorial

Álvarez del Blanco, M. (2010). Ampliación de la planta industrial de la Cervecería Boliviana Nacional SA (Doctoral dissertation)

Braidot, P. (2006). Neuromarketing: neuroeconomía y negocios. Nestor Braidot

De Andreis, A. (2012). Neuromarketing: una mirada a la mente del consumidor. Revista ADGNOSIS 
Doley, R. (2015). Brainfluence. 100 formas de convencer y persuadir a través del neuromarketing. Empresa Activa, España

Genco, S; Pohlmann, A; Steidl, P. (2013). Neuromarketing for dummies. John Wiley y Sons, Inc. Estados Unidos

González, Y., y Triana, D. (2018). Actitudes de los docentes frente a la inclusión de estudiantes con necesidades educativas especiales. Educación y Educadores

Kahneman, D. (2013). Thinking, Fast and Slow. Farrar, Straus and Giroux

Kumar, H., y Singh, P. (2015). Neuromarketing: An emerging tool of market research. International Journal of Engineering and Management Research

León, C. (2010). El neuromarketing: la llave de la caja de Pandora (Doctoral dissertation, Universidad del Rosario)

Manterola, C., y Otzen, T. (2015). Estudios experimentales 2 parte: estudios cuasiexperimentales. International Journal of Morphology
Millward, B (2009): Investigaciones realizadas para el Correo Real Británico (UK's Royal Mail). Centro Experimental de Psicología del Consumidor de la Universidad de Bangor

Monge, B., y Fernández, V. (2011). Neuromarketing: Tecnologías, Mercado y Reto.

Montterlini, M (2008): Economía emocional: En qué nos gastamos el dinero y por qué. Ediciones Paidós Ibérica. España

Renvoise, P; y Moryn, C. (2007). Neuromarketing. Editorial Thomas Nelson

Sandoval, C. (2019). Gestión por competencias en la cervecería SUREÑA Sociedad Anónima de la ciudad de Sucre. Universidad Andina Simón Bolívar. Bolivia

Torres, L. (2012). El Neuromarketing y la fidelización en los clientes de la Empresa Agroinsumos en la ciudad de Lasso provincia de Cotopaxi (Bachelor's thesis) 